\title{
Secondary Flow Control in Low Aspect Ratio Vanes Using Splitters
}

\author{
Christopher J. Clark* \\ Whittle Laboratory, \\ University of Cambridge, \\ $1 \mathrm{JJ}$ Thomson Avenue, \\ Cambridge CB3 ODY, UK \\ Email: cjc95@cam.ac.uk
}

\author{
Graham Pullan \\ Whittle Laboratory, \\ University of Cambridge, \\ $1 \mathrm{JJ}$ Thomson Avenue, \\ Cambridge CB3 ODY, UK
}

\author{
Eric Curtis \\ Whittle Laboratory, \\ University of Cambridge, \\ $1 \mathrm{JJ}$ Thomson Avenue, \\ Cambridge CB3 ODY, UK \\ Frederic Goenaga \\ Rolls Royce. \\ Bristol BS34 7QE, UK
}

\begin{abstract}
Low aspect ratio vanes, often the result of overall engine architecture constraints, create strong secondary flows and high endwall loss. In this paper, a splitter concept is demonstrated that reduces secondary flow strength and improves stage performance. An analytic conceptual study, corroborated by inviscid computations, shows that the total secondary kinetic energy of the secondary flow vortices is reduced when the number of passages is increased and, for a given number of vanes, when the inlet endwall boundary layer is evenly distributed between the passages. Viscous computations show that, for this to be achieved in a splitter configuration, the pressure-side leg of the low aspect ratio vane horseshoe vortex, must enter the adjacent passage (and not "jump" in front of the splitter leading edge).

For a target turbine application, four vane designs were produced using a multi-objective optimization approach. These designs represent: current practice for a low aspect ratio vane; a design exempt from thickness constraints; and two designs incorporating splitter vanes. Each geometry is tested experimentally, as a sector, within a low-speed turbine stage. The vane designs with splitters geometries were found to reduce the measured secondary kinetic energy, by up to $85 \%$, to a value similar to the design exempt from thickness constraints. The resulting flowfield was also more uniform in both the circumferential and radial directions. One splitter design was
\end{abstract}

*Address all correspondence to this author. 


\section{Introduction}

Engine architectures often force turbine designers to employ low aspect ratio stator vanes. Typical drivers for this suboptimal aerodynamic choice include the requirement to pass a structural component or oil pipe through the main gas path or to house instrumentation. Each of these requirements places a thickness constraint on the vane, leading to increased chord and hence low aspect ratio. Secondary flows are dominant in these vanes - there may be no discernible two-dimensional flow.

Several strategies have been demonstrated to improve the performance of stages with low aspect ratio vanes [1-3]. These include aft loading, contoured endwalls and unloaded endwall blade sections.

In this paper we will follow an alternative approach. On the assumption that the thickness constraint need not be applied to all the vanes in the row, the pitch-chord ratio of the low aspect ratio vanes is increased above the aerodynamic optimum and one or more high aspect ratio "splitter" vanes are added to the passage formed by adjacent thick vanes, Fig 1 . We will show that this concept has the following benefits: reduced secondary flow penetration, reduced exit secondary kinetic energy and greater pitchwise uniformity. These all contribute to an increase in stage efficiency.

Two examples of low aspect ratio vane rows with splitters have been reported. The first, at the Von-Karman Institute $[4,5]$, was a configuration designed by adding a non-loaded strut (low aspect ratio) to an existing stator row (high aspect ratio). Problems were encountered with incidence on to the short chord vanes due to the strut potential field. The second, at the University of Graz [6-8], took a previously designed low aspect ratio vane row and inserted splitter vanes at the rear of the passage. The splitter vanes were designed to intercept the secondary flows that had developed upstream due to the low aspect ratio strut, and straighten them to produce a more uniform exit flowfield.

While there are instances of low aspect ratio vane rows with splitters reported in the literature, our perspective is that a systematic exploration of the design space has not been described. In this paper, a combination of optimizer-driven CFD, and experimental testing in a turbine stage, is used to examine the loss generation in rows where the low and high aspect ratio vanes are designed together. This approach leads to the identification of critical flow features, and associated design recommendations, for vane rows employing splitters.

\section{The Splitter Vane Concept}

The role of splitter vanes is to reduce the vortex strength, loss, and spanwise extent, of secondary flow. The creation of secondary flow is an inviscid phenomenon. Vorticity present in the inlet boundary layer (normal to the flow) is distorted by the blade row such that it has a streamwise component at row exit. The streamwise vorticity downstream of the row may be approximated using Squire and Winter's result [9]:

$$
\xi=-2 \varepsilon \frac{\partial U}{\partial z}
$$

where, for a given row turning $(\varepsilon)$ and inlet boundary layer profile $\left(\frac{\partial U}{\partial z}\right)$, this equation suggests that the amount of streamwise vorticity $(\xi)$ at row exit is fixed. However, the designer has freedom to control the distribution of this vorticity.

The simplest distribution of exit streamwise vorticity is that where the total is divided into $n$ identical discrete vortices. Of interest is the loss caused by the mixing of these vortices. The loss can, to a first order, be assumed to scale with the kinetic energy of the vortex, the peak loss resulting from complete dissipation of the vortex.

A Rankine vortex with forced vortex core and free vortex outer is defined as:

$$
\begin{gathered}
V_{\theta}=\frac{\Gamma r_{v}}{2 \pi r_{v c}^{2}} \text { for } r_{v}<r_{v c} \\
V_{\theta}=\frac{\Gamma}{2 \pi r_{v}} \text { for } r_{v}>r_{v c} \\
K E_{\text {vortex }}=\int_{0}^{a r_{v c}} \frac{1}{2} \rho V_{\theta}^{2} 2 \pi r_{v} d r_{v}=\frac{\Gamma^{2} \rho}{4 \pi}\left(\ln (a)+\frac{1}{4}\right)
\end{gathered}
$$


where $r_{v}$ is the radius from the vortex centre, $r_{v c}$ is the radius of the vortex core and $\Gamma$ is the total circulation. An integral of the flow field between 0 and $a r_{v 1}, a$ being an arbitrary constant, shows that the total kinetic energy of a vortex scales with the circulation squared.

Equation 1 combined with Stokes' theorem states that not only is the streamwise vorticity fixed but also the total exit streamwise circulation. If the circulation is partitioned $n$ times, the total secondary kinetic energy is

$$
S K E=\sum_{n=1}^{n} K E_{\text {vortex }, n} \propto \sum_{n=1}^{n} \Gamma_{n}^{2}
$$

and, with reference to Equation (1), the sum of the circulation is constant

$$
\sum_{n=1}^{n} \Gamma_{n}=\mathrm{const}
$$

The total secondary kinetic energy scales with $1 / n$ with $n$ being the number of partitions of a given pitchwise length of inlet boundary layer. The dependence on $\Gamma_{n}^{2}$ means that to minimise $S K E$, peak circulation should be minimised. This is achieved, for a fixed number of partitions, by making all $\Gamma_{n}$ equal.

This result is supported with inviscid CFD. Figure 2 shows secondary kinetic energy coefficient as a function of $\frac{1}{A R}$ for an inviscid linear cascade. Each point used the same profile, performing $65^{\circ}$ of turning at midspan, whilst the vane chord and pitch are progressively reduced with a constant span and inlet boundary layer. The dependence on $\frac{1}{A R}$ is clear, this being equivalent to a change in vane count at constant pitch-to-chord ratio.

The aim of employing splitters is, therefore, to achieve an increased number of secondary flow vortices, each containing a smaller fraction of the total streamwise circulation. This will in turn reduce the total exit SKE. Equation 5 also shows that to minimise SKE for a given number of passages, $\Gamma_{n}$ should be equal for each passage.

\section{DESIRABLE FEATURES OF SPLITTER VANE DESIGN}

Before the particular turbine application and design methodology are described, and to aid discussion of the experimental and computational results, we describe two desirable features of splitter vane design.

\section{Horseshoe vortex migration}

When a vortex filament such as that in a boundary layer meets an obstacle in the flow, it becomes distorted around it causing the roll up of a horseshoe vortex. Classical secondary flow work has shown that, in the presence of a cross passage pressure gradient, one leg of the vortex migrates with the pressure gradient across the passage, with the remainder of the unseperated inlet boundary layer being entrained into it $[2,10]$. This entrainment increases the vorticity of the "passage vortex" and hence the secondary kinetic energy. The resulting vortex has a large fraction of the inlet boundary layer contained within its core.

We have seen that to minimise secondary kinetic energy the vortices at exit must have the same circulation. Therefore the trajectory of the horseshoe vortex becomes important (Fig. 3), particularly when the inclusion of different axial leading edge locations allows the possibility of the vortex passing, or "jumping", in front of the leading edge of a splitter vane. This would result in the "jumped" passage containing little of the inlet vorticity. Figure 3 shows a traditional secondary flow alongside a splitter arrangement with and without a "jump". The flow resulting from a jump consists of the majority of the inlet boundary layer fluid entering the second passage (P2) creating a vortex similar to a low aspect ratio design.

\section{Vane load matching}

Whilst the focus of this paper is to reduce secondary losses the final goal is to find the optimal balance between profile and secondary losses. For a turbulent boundary layer, Denton [11] proposes the the loss generated scales with the surface integral of $V^{3}$. This suggests that the minimum boundary layer loss is possible by reducing peak velocities. For a row with multiple blade geometries the " $V$ "argument" indicates that the peak velocities should be matched between vanes.

\section{TURBINE GEOMETRY \& TEST FACILITY}

The turbine used in the current work is a low-speed single stage test facility at the Whittle Laboratory. The turbine has low aspect ratio vanes and is representative of the Intermediate Pressure turbine of a three shaft aero engine, or the first stage of the Low Pressure turbine in a two shaft architecture. 
Figure 4 shows a meridional view of the turbine. The facility, most recently reported by [12], is full scale $\left(r_{t i p}=0.55 \mathrm{~m}\right)$ and low speed $\left(M_{2}=0.25\right)$. The low aspect ratio NGVs Reynolds numbers, based on mid-span chord are $5.3 \times 10^{5}$, the rotor Reynolds number is $1.6 \times 10^{5}$. The rotor is shrouded and sealed against the axial face of the shroud, reducing the influence of radial thermal expansion on the leakage gap. There are representative hub cavities and rim seals both upstream and downstream of the NGV row with, for the present study, zero net leakage. The inlet conditions are set by a turbulence gauze and endwall turbulators to produce a representative total pressure profile. A row of inlet guide vanes fix the zero-swirl inlet condition.

The majority of data presented in this paper were measured with a 5-hole pneumatic probe (probe head diameter of $2.05 \mathrm{~mm}$ ) with a spatial resolution of approximately $2.5 \mathrm{~mm}$ ( $4 \%$ of NGV exit span) in the pitchwise and spanwise directions. The traverses were performed behind a $90^{\circ}$ sector of each new geometry and periodicity was obtained for over half of the sector in each case. To avoid the endwalls influencing the probe, half a probe diameter, $1 \mathrm{~mm}$, clearance is left between the traverse and the endwall.

When a full ring of the same NGV design is installed (not just a $90^{\circ}$ sector), efficiency can be calculated from measurements of brake power output, mass flow, inlet total temperature and total pressure at inlet and exit of the stage. Power output is derived from a load cell attached to the eddy-current dynamometer that controls the shaft speed. The mass flow is found from a calibrated intake contraction which also houses 8 thermocouples to obtain the inlet total temperature. Inlet and exit total pressures are measured by 10 Pitot probes located around the annulus at differing spanwise locations; these Pitots are traversed in a pitchwise direction to ensure that the inlet guide vane wakes at inlet, and remaining NGV structures after the rotor, are captured. The use of multiple fixed probes allows simultaneous measurements around the annulus providing an average over multiple passages. Humidity and atmospheric pressure variations during a run are measured and the properties of air adjusted accordingly. The efficiency tests are performed at constant NGV Reynolds number, with the blade speed varying to suit. Experience on the turbine and continual improvements have shown that the measurements are now repeatable to within $\pm 0.1 \%$ of brake efficiency $(\eta)$.

\section{DESIGN METHODOLOGY}

A row of non-uniform vanes increases the design space within which the designer must seek an optimum. Each splitter vane adds to the total number of parameters required to define the geometry. In addition, the designer will have a clear understanding of the influence of some parameters in a uniform blade row but have little or no experience of changing the same parameter in a row comprised of different vane geometries. As an example, Fig. 5 shows that increasing the stagger of one vane in a non-uniform row closes one throat and opens another rather than closing both as in a uniform row.

As well as the continuous parameters that define profile shape, there is a group of discrete parameters that define the topology of the design (for example, number of low aspect ratio vanes and the number of splitters). These cause discontinuities in the design space.

The strategy adopted was to fix the vane and splitter counts for a series of designs and use an optimizer to develop the profile shapes for each of these topologies. One advantage of using an optimizer, rather than manual iteration, is that each design is developed using the same process rather than later designs benefiting from the experience gained on earlier topologies. The vane counts of the four NGV designs are shown in Table 1. NGV1 represents the current practice comprising low aspect ratio vanes only. NGV2 represents a design exempt from the thickness constraints.

The optimization process is a multi-objective implementation of the TABU algorithm [13-16]. The geometry constraints are outlined in Table 2. The peak thickness constraint represents the need to pass a cylinder of diameter 37.5\% of NGV1's meridional chord for the low aspect ratio vanes. NGV2, representing a design in the absence of service constraints, was designed using the splitter vane limits. Each design was optimized to maximise stage efficiency whilst producing the same stage loading with the same rotor. The performance was evaluated using steady RANS CFD using the Turbostream [17] GPU accelerated solver. The interface between NGV and rotor was modelled using a mixing plane and the turbulence with the Spallart Almaras model [18]. The blades were meshed using a C-H topology with flexible interfaces between adjacent blades, NGV1 containing 300,000 cells, the more complicated NGV4 requiring 650,000. A relatively coarse boundary layer mesh $\left(y^{+} \simeq 30\right)$ was used with wall functions enabling a solution to be evaluated in 7 minutes on two GPUs. This speed of computation enabled a total of over $3000 \mathrm{NGV}$ designs to be evaluated in each case.

Due to the requirement to design multiple vanes in each row simultaneously a low parameter count per vane was desired. The parameters used to define each section are outlined in Fig. 6. These were combined to generate a profile using shape space to ensure continuous curvature [19-21]. To allow three-dimensional vane shapes, whilst keeping low parameter counts, the vanes were designed on three sections; hub, mid-span and casing. This still gives 90 parameters for NGV4.

The trailing edge location in the meridional view was constant for both low aspect ratio and splitter vanes in all designs. The reasoning for this choice was two-fold. The clear benefit of aft-loaded NGVs [1] forms a strong argument for splitter vanes to be located at the rear of the passage. If the velocities and geometry at the rear of both vanes are similar, as expected, there is no reason to extend one past the other. Finally the low aspect ratio vane leading edges were also fixed as a shorter design would lead to excessive thickness to chord ratios. 
The optimization was configured to minimise the stage lost efficiency $\left(\eta_{\text {lost }}\right)$ whilst maintaining stage loading ( $\psi$ ) within $\pm 2.5 \%$ at the same flow coefficient $(\phi)$. Each NGV was designed to be compatible with the same rotor. The use of the same rotor helped to bound the problem, but it is likely that the uniform flowfield generated by some of the splitter designs would allow an improved stage performance to be obtained from a re-designed rotor.

\section{NGV DESIGNS AND TEST RESULTS \\ Loading distributions}

The mid-span geometries of the four final designs are shown in Fig. 7, and their mid-span pressure distributions in Fig. 8. The pressure profiles show NGV1 in black for reference in each case. The straight cylinder passing through the low aspect ratio vanes limited the amount of lean possible, the splitter vanes similarly having little 3D stacking. NGV2, exempt from passing a cylinder, features compound lean.

All of the pressure distributions are aft-loaded, however the low aspect ratio vanes of NGV3 and NGV4 show progressively reduced loading at the leading edge of the vane (NGV4 has negative loading over the first $25 \%$ of meridional chord). It will be shown that this loading profile controls the development of the horseshoe vortex formed at the low aspect ratio vane leading edge and, in particular, prevents it from passing in front of the adjacent splitter vane.

Both splitter designs (NGV3 and NGV4) show matched peak velocities between vanes in agreement with the $V^{3}$ argument presented previously. The reduction in loading of NGV4 will be shown to be due to controlling the inlet boundary layer.

\section{Flowfield}

The flow visualisation presented in Fig. 9 shows, qualitatively, that the design intent of reducing secondary flow size has been met. The green dye was seeded on the upstream end walls (hub and casing) and its path across the vane suction surface shows the lift off line associated with the passage vortices. The spanwise penetration of the passage vortices away from the endwalls, particularly the casing vortex, has been reduced significantly. NGV1 exhibits the largest vortex penetration with the casing vortex covering $55 \%$ of the span from the casing. NGV2 shows a penetration of $22 \%$, suggesting a $50 \%$ reduction in vortex spanwise extent. The passage vortices of NGV3 move $30 \%$ of span from the casing on both the low aspect ratio vane and the splitter vane, (the increase relative to NGV2 can be accounted for by the reduction in total vane count from 56 to 42). For NGV4, the casing passage vortex penetration is $18 \%$ of span for the low aspect ratio vane, the splitter adjacent to the large vane's pressure surface has a vortex penetration of $29 \%$ and the final splitter $18 \%$. This indicates that whilst being successful in partitioning the inlet vorticity three ways, the NGV4 design has resulted in passage vortices of unequal size.

A view of the hub from the same series of flow visualisation experiments is presented in Fig. 10. Red dye from the blade surface has recirculated to the horseshoe vortex lift-off line in both cases showing the extent of the vortex. The lift-off lines associated with the passage vortices pass into the adjacent passage in both instances, avoiding vortex jumping. Moreover, the trajectory of the pressure-side leg of the low aspect ratio vane's horseshoe vortex can be seen to remain close to its pressure surface in both NGV3 and NGV4 due to the aft-loading of the low aspect ratio vane. The proximity of the horseshoe vortex to the splitter leading edge suggests that, without the low leading edge loading of the low aspect ratio vane, the horseshoe vortex would perform a vortex "jump" as previously described.

Taken together, Figs. 9 and 10 suggest that there is a minimum passage vortex size possible, for a given inlet boundary layer, with the low aspect ratio vane present. The low aspect ratio vane of NGV4 has a pressure distribution that diverts as much inlet vorticity into the suction-side leg of its horseshoe vortex as possible (it has minimal loading prior to the splitter passage). The addition of another splitter vane would likely force the vortex to jump passages.

The casing flow visualisation displays the same topology for all cases as that on the hub with a single pressure-side vortex leg entering each passage.

Figure 11 shows the meridional yaw angles at vane row exit. The flow angle contributions due to the vortical structures are evident, vortices appear on yaw angle contours as closely spaced horizontal contours representing the core followed by elliptic contours in the free vortex exterior, the most prominent being the casing passage vortex (PV) and adjacent shed vortex (SV) [22]. Whilst NGV3 has reduced pitchwise variations it still has $10^{\circ}$ of endwall overturning. Both NGV2 and NGV4 reduce this overturning to produce a much more uniform flow angle distribution. The flow angle variations (over and under turning) caused by the two passage vortices present behind NGV3 are very similar. This suggests that both vortices have the same circulation, both reduced compared to that of NGV1, this is in agreement with the preceding analytic arguments.

The magnitudes of the vortices in NGV4 make them hard to discern, with only the first splitter showing a structure similar to NGV2. This agrees with the flow visualisation and provides confirmation that streamline penetration depth is a good indicator of vortex size.

The pitchwise averaged yaw angle distributions are presented in Fig. 12. The distributions can be grouped into two sets: NGV1 and NGV3; and NGV2 and NGV4. For the latter set, the casing overturning is present in only the outer 20\% of span, reduced from $30-35 \%$ for NGV1 and NGV3. The hub overturning is almost completely absent for NGV2 and 
NGV4, compared to the $15-20 \%$ of span for NGV1 and NGV3. This confirms that splitter vanes can reduce the overturning associated with secondary flows, in the same manner as a high aspect ratio row.

The swirl velocity distributions can be integrated to confirm that each of the four designs is performing the same duty. The sector experimental tests do not enable the stage loading, held constant in the optimization, to be evaluated. The most relevant measurable quantity being the rotor inlet angular momentum,

$$
M_{t}=\frac{\int r V_{t} \mathrm{~d} \dot{m}}{r_{m i d} \overline{\overline{V_{m}}} \dot{m}}
$$

which we have non-dimensionalised here. The double over-bar represents the passage averaged value. The values of $M_{t}$ for each of the four designs are listed in Table 3. It can be seen that the rotor inlet angular momentum has changed by less than $4 \%$, within the tolerance set for the optimization, equivalent to an average exit angle change of $0.7^{\circ}$.

The exit flow angle differences between the designs correlate with changes to the spanwise distribution of mass flow. Figure 13 shows the meridional flow coefficient based on rotor speed at mid-span. The reduction of the overturning has made the distribution of mass flow more uniform. The benefits of this to subsequent rows are multiple: a uniform mass flow distribution allows for uniform blade loading along the span, in turn leading to reduced blade velocities and, by considering a $V^{3}$ argument, lower losses.

The streamwise vortical structures seen in the exit flowfield of the vane row are, by definition, associated with velocity components normal to the bulk flow vector. As these vortical structures are expected to mix out in the interstage gap and following rows, this kinetic energy will be dissipated. To quantify this, a secondary kinetic energy is used

$$
\begin{gathered}
\underline{n_{b u l k}}(r)=\frac{\left[\overline{V_{x}}, \overline{V_{r}}, \overline{V_{t}}\right]}{\overline{V_{a b s}}}, \quad \underline{V_{\text {sec }}}=\left(\underline{V} \times \underline{n_{\text {bulk }}}\right) \\
C_{S K E}=\frac{K E_{\text {sec }}}{K E_{\text {bulk }}}=\frac{\frac{1}{2} \rho\left(\underline{V_{\text {sec }}} \cdot \underline{V_{\text {sec }}}\right)}{\frac{1}{2} \rho\left({\overline{\bar{V}_{x}}}^{2}+{\overline{\bar{V}_{r}}}^{2}+\overline{\bar{V}}^{2}\right)}
\end{gathered}
$$

where single overbars denote the pitchwise average at that spanwise position and double overbars an average over the entire passage.

The spanwise distributions in Fig. 14 show the kinetic energy associated with the secondary velocity components contained within the vortical structures. The reduction from NGV1 to the other designs is evident, see also Table. 3, as is the reduction in penetration depth.

To summarize the changes in flowfield, all designs show improvements in both endwall overturning, and pitchwise uniformity, when compared to NGV1. The exit flowfield of NGV4 reproduces the exit flowfield of NGV2 whilst retaining a low aspect ratio vane and enabling the passing of services.

\section{Loss}

Due to the distortions of the axi-symmetric stream surfaces passing through the passage, removing the effect of the inlet profile is non-trivial. The coefficients presented are based on the average inlet total pressure and hence represent redistribution of the inlet profile as well as total pressure drop within the row. When averaged over the whole passage this becomes a loss coefficient,

$$
Y_{p}=\frac{\overline{\overline{P_{0,1}}}-P_{0}}{\frac{1}{2} \rho\left({\overline{\overline{V_{m}}}}^{2}+{\overline{\overline{V_{t}}}}^{2}\right)}
$$

The contours of stagnation pressure coefficient in Fig. 15 show the wake structures of each design. The spanwise extent of the "2D" region of the wake is only $25 \%$ of span in NGV1 compared to $62 \%$ in NGV 2. The passage vortices distort the wake close to the casing, their interaction with the blade surface results in the sweep of the suction surface boundary layer away from the casing, to the lift-off line of the vortex. 
The spanwise stagnation pressure distributions in Fig. 16 allow for a more quantitative analysis. The largest difference on the plot is the elevated total pressure drop in the two-dimensional region of NGV1. The cross-span sweep of the secondary flows confines NGV1's vane surface boundary layer so that it has a similar loss to that found in the vortex. The spanwise extent of the $2 \mathrm{D}$ region is raised for NGV2 and NGV4, with the profile of NGV4 closely resembling the inlet total pressure profile.

Mixing losses, calculated using a mixing-plane calculation, conserving mass, axial, radial and tangential momentum as well as total temperature at each spanwise location,

$$
\overline{Y_{p, \text { mix }}}=\frac{\overline{P_{0,2}}-\overline{P_{0, \text { mixed }}}}{\frac{1}{2} \rho\left({\overline{\overline{V_{m}}}}^{2}+{\overline{\overline{V_{t}}}}^{2}\right)}
$$

are shown as a distribution in Fig.17. The mixing losses show peaks clearly associated with each of the vortical structures previously highlighted. This again confirms the reduction in penetration depth and vortex strength, however this result has a direct implication for NGV performance and loss generation.

The comparison between SKE, Fig. 14 and mixing loss, shows not only that peak locations are very well aligned, but also that the values along the span are almost equal. This suggests that the mixing loss seen is due almost entirely to the SKE.

Table 3 shows the average performance metrics of each NGV. The passage loss coefficients show a reduction in loss for both NGV2 and NGV3 compared to NGV1. NGV4, however, has a rise in passage loss, this is likely due to the increased surface area and trailing edge count.

The mixing loss reduces by over $80 \%$ for NGV4. The ranking of designs based on mixing loss aligns with the total number of passages in agreement with the earlier analytic arguments. The total loss including both sources show a reduction of $15.3 \%$ for NGV3, being very close to NGV2's reduction of $15.6 \%$. NGV4's reduction in mixing loss outweighs its increase in passage loss, still leading to a $9.6 \%$ reduction in total loss.

\section{Stage Efficiencies}

The sector tests used previously were unable to directly measure efficiency as this requires shaft torque measurements. Full rings of both NGV1 and NGV3 were tested for stage performance.

The stage loading between the two designs matched at the design point to within $1 \%$. The normalised efficiency characteristics are shown in Fig. 18, $\eta_{\text {ref }}$ is the stage efficiency of NGV1 at the design flow coefficient. The improvement of NGV3 over NGV1 is clear, at the design point the improvement was measured to be $0.88 \%$ stage efficiency.

Combining the NGV exit traverse results with the efficiency results the efficiency improvements can be split up as shown in Table. 4

This makes it clear that the single largest contribution comes from the reduction of mixing out of the secondary kinetic energy.

\section{RETURN TO THE SPLITTER VANE CONCEPT}

The performance improvements demonstrated in this paper have been attributed to a reduction in secondary flow strength. It has been shown that this is due to the partitioning of the streamwise vorticity. Although not exhibited in the designs presented here, it is anticipated that the pressure side leg of the low aspect ratio vane horseshoe vortex could migrate in front of the leading edge of a splitter vane. This would redistribute inlet boundary layer fluid, and the associated vorticity, from the "jumped" passage into the passage that the pressure-side leg now enters.

To explore this phenomenon, CFD results are included of NGV4 (the design that computed streamlines suggest is closest to exhibiting a vortex jump) as well as two modified geometries. The modifications progressively reduce the chord of the splitter adjacent to the low aspect ratio vane's pressure surface in 5\% increments at all spanwise locations.

Figure 19 shows the casing streamlines for the three geometries. The original geometry, Fig. 19 (a), shows the lift-off line associated with the low aspect ratio vane's pressure-side leg, labelled PS leg 1, pass into passage 1 . The inlet boundary layer below this line reaches the splitter between passage 1 and 2 , it then rolls up to form a second horseshoe vortex, its pressure-side leg (PS leg 2) passing into passage 2. Tracing the streamlines upstream, the inlet boundary layer can be seen to have fluid entering both passages.

The geometry with 5\% reduced chord, Fig. 19 (b), shows the lift-off line pass the splitter pressure surface. This topology shift prevents a distinguishable second horseshoe vortex forming. As the lift-off line occurs at the outermost edge of the vortex its core impinges onto the splitter. This results in redistribution of inlet boundary layer from passage 1 to passage 2 and large incidence variation onto the splitter. The streamline stagnating on the splitter between passage 1 and 2 can be seen to approach with roughly $35^{\circ}$ of incidence rather than the $-5^{\circ}$ seen in Fig. 19 (a). 
The final geometry, $-10 \%$ meridional chord, Fig. 19 (c), shows the vortex passing the leading edge and being caught by the next passage's pressure gradient sweeping rapidly over to the second splitter's suction surface. In this situation very little inlet boundary layer fluid enters passage 1, however passage 2 now receives much more than the original geometry. The incidence onto the splitter is severe with the flow angle being roughly $45^{\circ}$.

These streamline positions show that the inlet boundary split between the passages has changed. The horseshoe vortex pressure-side leg "jump" has resulted in a less uniform partition of inlet boundary layer between the passages.

The effect on the NGV exit flow field is presented in Fig. 20 and Fig. 21. The increase in inlet boundary layer located in the second splitter's vortex core is evident as an increase in its size and total pressure deficit. The reduction of the loss core from splitter 1 shows that it is a redistribution rather than change in total deficit. The change in flow angles show the increase in the strength of the vortex pair leaving passage 2 .

The secondary kinetic energy for the 3 geometries is presented in Fig. 22. The SKE located within both the vortical structures can be seen to rise as the splitter chord is reduced. The predicted SKE coefficients are presented in Table 5. The full jump can be seen to cause in increase in SKE of 61.9\%.

The role of the low aspect ratio vane horseshoe vortex has been explore. The finding is that the migration of the pressure-side leg of the low aspect ratio vane horseshoe vortex upstream of a splitter vane has the potential to cause an uneven distribution of inlet boundary layer. This leads to uneven exit streamwise vorticity and an increase in exit secondary kinetic energy as well as causing an increase in flow non-uniformity. It is concluded that the migration of the pressure surface leg of the low aspect ratio horseshoe vortex in front of a splitter should be avoided.

\section{CONCLUSIONS}

The conclusions we draw are divided into three sections:

1. The splitter concept has been shown to control the secondary flows in a row with low aspect ratio vanes. The rationale behind the concept is to reduce the secondary mixing losses. Reduced endwall loss, particularly from the mixing-out of secondary flow vortices, is obtained if the total secondary vorticity is subdivided into many vortices. Both a simplified analysis, and inviscid computations, show that the loss obtained from mixing secondary flows is proportional to the inverse of the row aspect ratio. In rows where low aspect ratio vanes are required, splitters can be employed to increase the average aspect ratio. Further, it has been shown that for a given total number of vanes (low aspect ratio and splitters) the lowest secondary flow mixing loss is obtained when the exit streamwise vorticity is distributed evenly across the passages, i.e. the secondary kinetic energy of each passage is the same.

2. An optimizer-driven process was used to design the low aspect ratio vane and splitters together. Two over-riding design principles were found. First, in order to develop similar secondary flows at the trailing edge of each vane, the vorticity in the inlet endwall boundary layer should be partitioned evenly across each passage. This means that the trajectory of the leading edge horseshoe vortices, particularly that of the pressure-side leg of the horseshoe vortex of the low aspect ratio vane, must be controlled by careful positioning of the splitters and the vane potential fields. "Jumping" of the pressure-side leg of the low aspect ratio vane in front of the adjacent splitter should be avoided. Secondly, profile loss minimisation between non-uniform vanes is achieved by matching peak velocities of the vanes (low aspect ratio and splitter). This was evident in both of the optimized splitter designs.

3. For the particular test turbine under consideration, a splitter design was able to reduce the NGV row loss by $6.9 \%$. The same splitter design was able to reduce the NGV exit mixing loss by $77.4 \%$. Finally, this design was found to have a stage efficiency improvement of $0.88 \%$ compared to the datum design.

\section{Acknowledgements}

The authors would like to thank Nick Hooper of the Whittle Laboratory as well as Simon Bather and Mark Stokes of Rolls-Royce for their advice and technical expertise throughout this work. The work was funded by Rolls-Royce plc under the SILOET 2 work package.
Nomenclature
$H$ Span
$C$ Chord
$S$ Pitch
$\alpha$ Yaw angle
$\beta$ Pitch angle 
$M_{t} \quad$ Angular momentum coefficient

$\xi \quad$ Streamwise vorticity

$\Gamma$ Circulation

$C_{S K E} \quad$ Ratio of secondary to bulk kinetic energy $=\frac{S K E}{K E_{b u l k}}$

$Y_{p}$ Total pressure coefficient

$Y_{p, m i x}$ Mixing total pressure coefficient

$C_{p} \quad$ Static pressure coefficient

$\phi$ Flow coefficient

$\psi \quad$ Stage loading

$\eta \quad$ Stage efficiency

$\eta_{\text {lost }}$ Lost stage efficiency

[]$_{m}$ Meridional value

[]x Axial value

[] $r$ Radial value

[] $t$ Tangential value

[]o Stagnation value

[1 1 At NGV inlet

[]$_{2}$ At NGV exit

[ $]_{3}$ At rotor exit

[] $]_{\text {sec }}$ Normal to primary vector

[] bulk Aligned with primary vector

KE Kinetic energy

SKE Secondary kinetic energy

LE Leading edge

TE Trailing edge

NGV Nozzle guide vane

AR Aspect ratio

$\bar{x} \quad$ Pitchwise average of $\mathrm{x}$ at a spanwise location

$\overline{\bar{x}} \quad$ Passage wide average of $\mathrm{x}$

\section{References}

[1] Pullan, G., Denton, J., and Curtis, E., 2006. "Improving the Performance of a Turbine With Low Aspect Ratio Stators by Aft-Loading". ASME Journal of Turbomachinery, 128, pp. 492-499.

[2] Langston, L. S., 2001. "Secondary flows in axial turbines-a review.". Annals of the New York Academy of Sciences, 934, pp. 11-26.

[3] Dunkley, M. J., 1998. “The Aerodynamics of Intermediate Pressure Turbines”. PhD thesis, University of Cambridge.

[4] Lavagnoli, S., Yasa, T., Paniagua, G., Castillon, L., and Duni, S., 2012. "Aerodynamic Analysis of an Innovative Low Pressure Vane Placed in an s-Shape Duct”. ASME Journal of Turbomachinery, 134, pp. 011013-1-011013-13.

[5] Yasa, T., Lavagnoli, S., and Paniagua, G., 2011. "Impact of a multi-splitter vane configuration on the losses in a 1.5 turbine stage". Proceedings of the Institution of Mechanical Engineers, Part A: Journal of Power and Energy, 225.

[6] Spataro, R., Santner, C., Lengani, D., and Göttlich, E., 2012. "On the flow evolution through a lp turbine with widechord vanes in an s-shaped channel". ASME Turbo Expo GT2012-681.

[7] Spataro, R., Göttlich, E., Santner, C., and Heitmeir, F., 2014. "A numerical comparisonon the aerodynamic performances of a two-stage two-spool turbine facility predicted by steady and unsteady simulations". In 10th European Conference on Turbomachinery Fluid Dynamics and Thermodynamics, ETC 2013.

[8] Spataro, R., Göttlich, E., Lengani, D., Faustmann, C., and Heitmeir, F., 2014. "Development of a Turning Mid Turbine Frame With Embedded DesignPart I: Design and Steady Measurements". ASME Journal of Turbomachinery, 136, pp. 071008-1-071008-9.

[9] Squire, H. B., and Winter, K. G., 1951. "The Secondary Flow in Cascade of Aerofoils in Non-Uniform Stream". Journal of Aeronautical Science, 18, pp. 271-277.

[10] Langston, L. S., Nice, M. L., and Hooper, R. M., 1977. "Three-Dimensional Flow Within a Turbine Cascade Passage”. Journal of Engineering for Power, 99, pp. 21-28.

[11] Denton, J. D., 1993. "The 1993 IGTI Scholar Lecture: Loss Mechanisms in Turbomachines”. ASME Journal of Turbomachinery, 115.

[12] Cranstone, A. W., Pullan, G., Curtis, E. M., and Bather, S., 2012. “Aerodynamic Design of High Endwall Angle Turbine Stages: Part 1Methodology Development”. ASME Turbo Expo GT2012-68705, pp. 1179-1189.

[13] Glover, F., 1989. “Tabu Search - Part I”. ORSA Journal on Computing, 1, pp. 190-206. 
[14] Jaeggi, D. M., Parks, G. T., Kipouros, T., and Clarkson, P. J., 2008. "The development of a multi-objective Tabu Search algorithm for continuous optimisation problems". European Journal of Operational Research, 185, pp. 1192-1212.

[15] Jaeggi, D., Parks, G., Kipouros, T., and Clarkson, J., 2005. "A Multi-objective Tabu Search Algorithm for Constrained Optimisation Problems". Lecture Notes in Computer Science, 3410, pp. 490-504.

[16] Jaeggi, D., Asselin-miller, C., Parks, G., Kipouros, T., Bell, T., and Clarkson, J., 2004. "Multi-objective Parallel Tabu Search". pp. 732-741.

[17] Brandvik, T., and Pullan, G., 2011. "An Accelerated 3D NavierStokes Solver for Flows in Turbomachines". ASME Journal of Turbomachinery, 133, pp. 021025-1-021025-9.

[18] Spalart, P. R., and Allmaras, S. R., 1994. "A one-equation turbulence model for aerodynamic flows". La Recherche Aerospatiale, 1, pp. 5-21.

[19] Kulfan, B. M., 2008. "Universal Parametric Geometry Representation Method". Journal of Aircraft, 45, pp. 142-158.

[20] Kulfan, B. M., 2007. "Recent Extensions and Applications of the CST Universal Parametric Geometry Representation Method". Technology.

[21] Kulfan, B. M., and Bussoletti, J. E., 2006. “"Fundamental” parametric geometry representations for aircraft component shapes". Collection of Technical Papers - 11 th AIAA/ISSMO Multidisciplinary Analysis and Optimization Conference, 1, pp. 547-591.

[22] Pullan, G., Denton, J., and Dunkley, M., 2003. "An Experimental and Computational Study of the Formation of a Streamwise Shed Vortex in a Turbine Stage". ASME Journal of Turbomachinery, 125, pp. 291-297. 
Table 1: NGV design topologies

\begin{tabular}{lll}
\hline Design & Low aspect ratio count & Splitter count \\
\hline NGV1 & 28 & 0 \\
NGV2 & 0 & 56 \\
NGV3 & 21 & 21 \\
NGV4 & 21 & 42 \\
\hline
\end{tabular}


Table 2: NGV geometric constraints

\begin{tabular}{cll}
\hline Feature & Low aspect ratio vane & Splitter vane \\
\hline$\frac{R_{l e}}{C_{m, N G V 1}}$ & $>6 \%$ & $>3 \%$ \\
$\beta_{t e}$ & $>8^{\circ}$ & $>8^{\circ}$ \\
$\frac{D_{t e}}{C_{m, N G V 1}}$ & $1.25 \%$ & $1.25 \%$ \\
$\frac{t_{\max }}{C_{m, N G V 1}}$ & must pass cylinder of $37.5 \%$ & $>6 \%$ \\
\hline
\end{tabular}


Table 3: NGV experimental performance

\begin{tabular}{lllllll}
\hline Design & $\phi_{m}$ & $M_{t}$ & $\overline{\overline{C_{s k e}}}$ & $\overline{\overline{Y_{p}}}$ & $\overline{\overline{Y_{p, \text { mix }}}}$, & $\overline{\overline{Y_{p, \text { total }}}}$ \\
\hline NGV1 & 0.514 & 2.585 & 0.84 & 6.67 & 1.06 & 7.72 \\
NGV2 & 0.514 & 2.682 & 0.19 & 6.27 & 0.24 & 6.51 \\
NGV3 & 0.514 & 2.581 & 0.21 & 6.21 & 0.33 & 6.54 \\
NGV4 & 0.514 & 2.643 & 0.12 & 6.81 & 0.17 & 6.98 \\
\hline
\end{tabular}


Table 4: Experimentally derived lost efficiency break down

\begin{tabular}{cl}
\hline Loss source & $\Delta \eta_{\text {lost }}=\eta_{\text {lost }, N G V 3}-\eta_{\text {lost }, N G V 1}$ \\
\hline NGV & $-0.25 \%$ \\
Mixing & $-0.46 \%$ \\
Rotor & $-0.17 \%$ \\
\hline
\end{tabular}


Table 5: $C_{S K E}$ of adjusted NGV4 geometries

\begin{tabular}{cl}
\hline \multirow{2}{*}{$C_{x}$ reduction } & $\frac{\overline{\overline{C_{S K E}}}}{\overline{\overline{C_{S K E}}}, N G V 4}$ \\
\hline $0 \%$ & $100.0 \%$ \\
$5 \%$ & $133.0 \%$ \\
$10 \%$ & $161.9 \%$
\end{tabular}




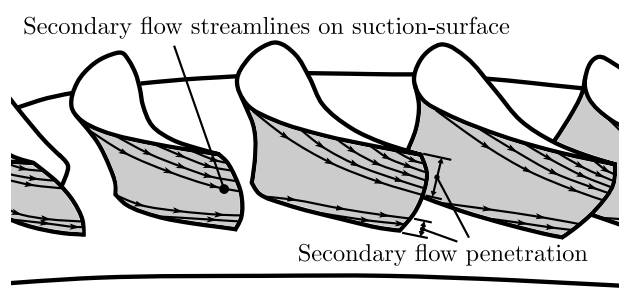

(a) Uniform low aspect ratio vanes Low aspect ratio vane

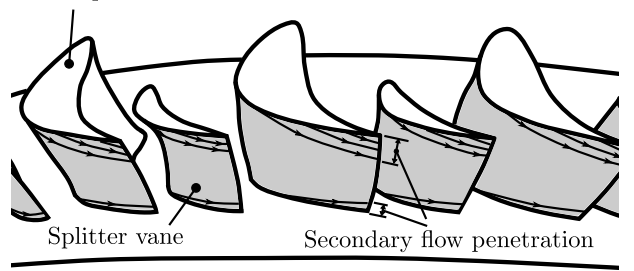

(b) Splitter concept (one splitter per low aspect ratio vane)

Fig. 1: Comparison of a uniform low aspect ratio vane row and splitter vane concept highlighting the reduced secondary flow penetration achieved by the splitter concept 


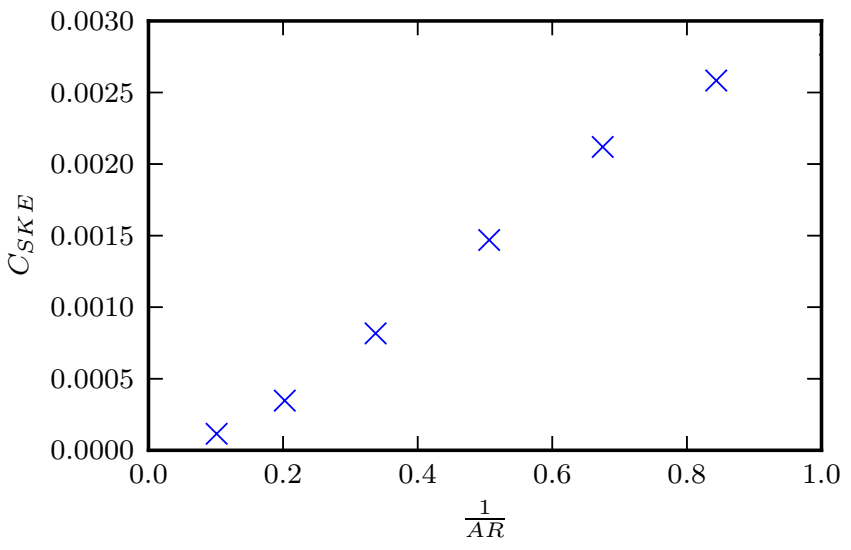

Fig. 2: Results for an inviscid blade row computation. Exit secondary kinetic energy, $C_{S K E}$, reduces as aspect ratio (AR) increases 


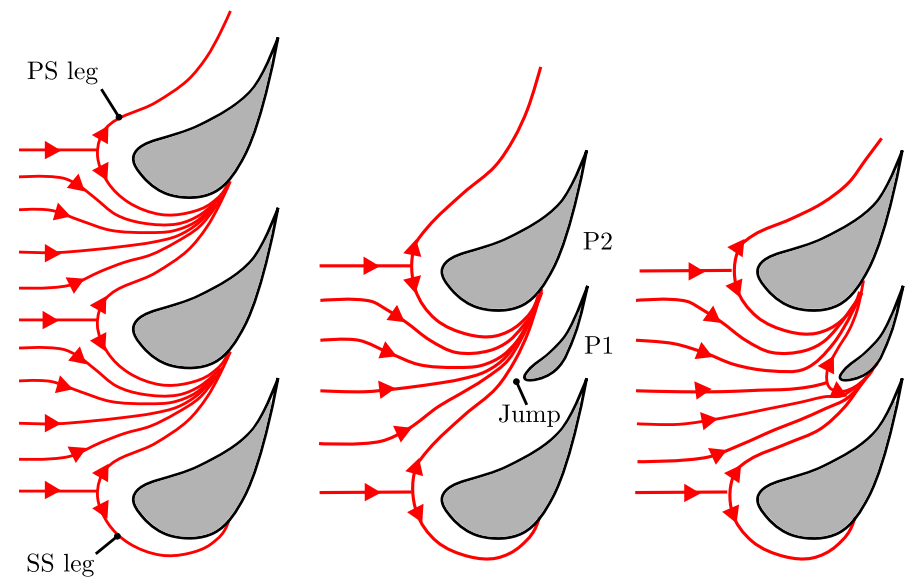

Fig. 3: Streamlines in the inlet endwall boundary layer to illustrate traditional secondary flow (left) and splitter secondary flows with (middle) and without (right) a vortex "jump" 


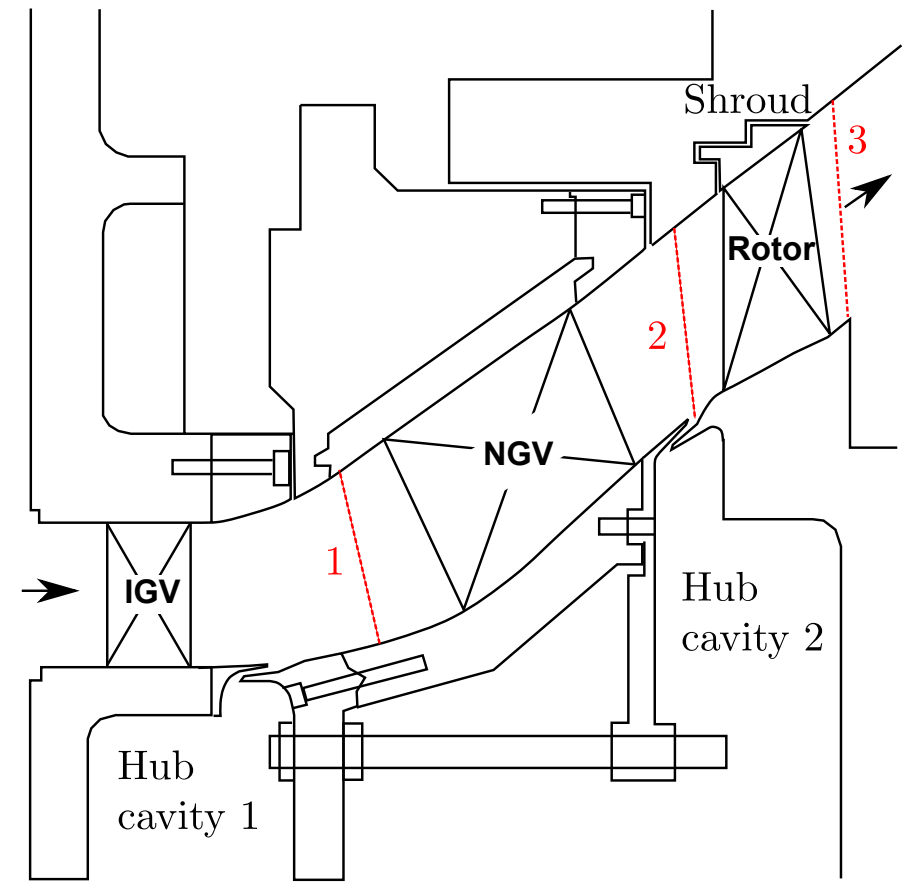

Fig. 4: Meridional view of the test facility showing traverse planes 

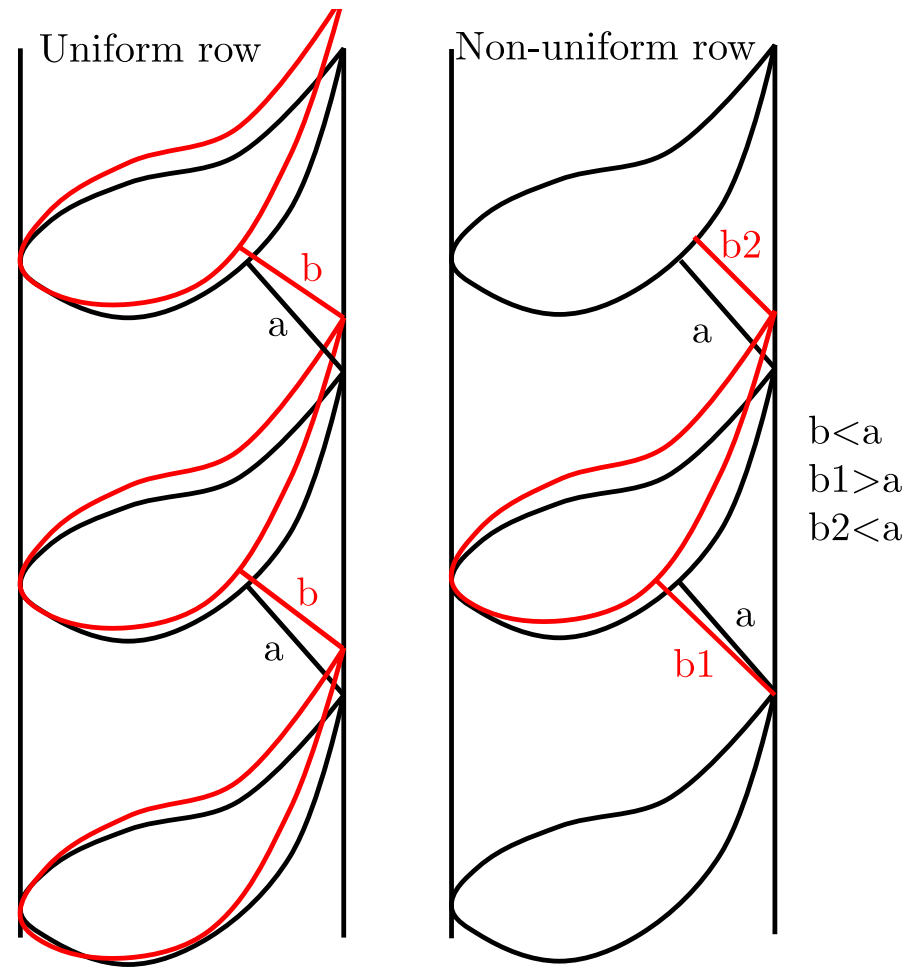

Fig. 5: Schematic demonstrating how a changing a conventional variable (stagger) causes a different effect for a non-uniform row compared to a uniform row, in this case causing some throats to open and others to close rather than a uniform closing of throats 


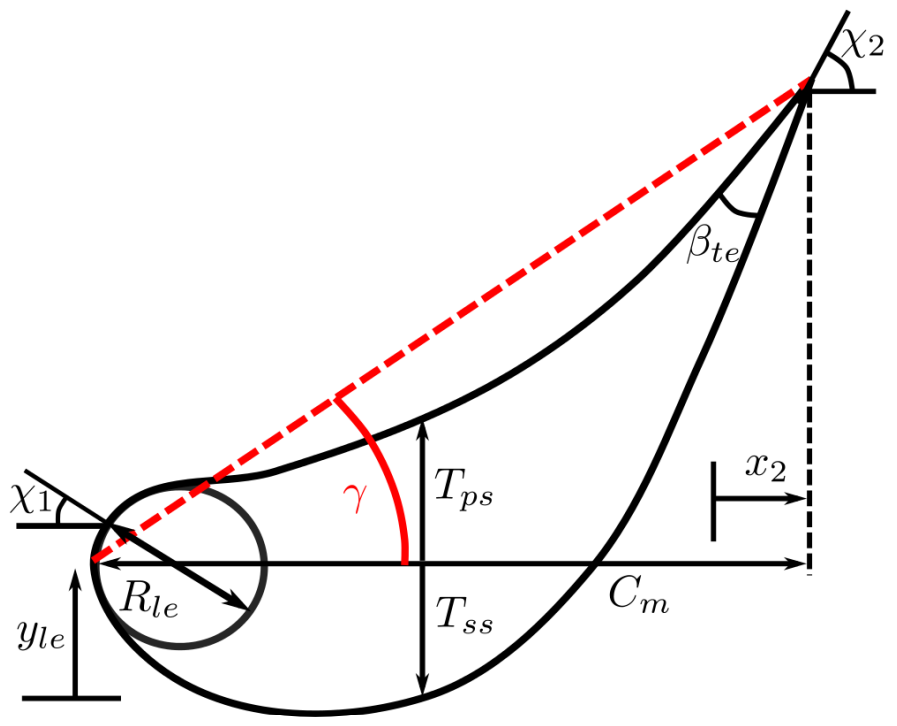

Fig. 6: Blade definition parameters 

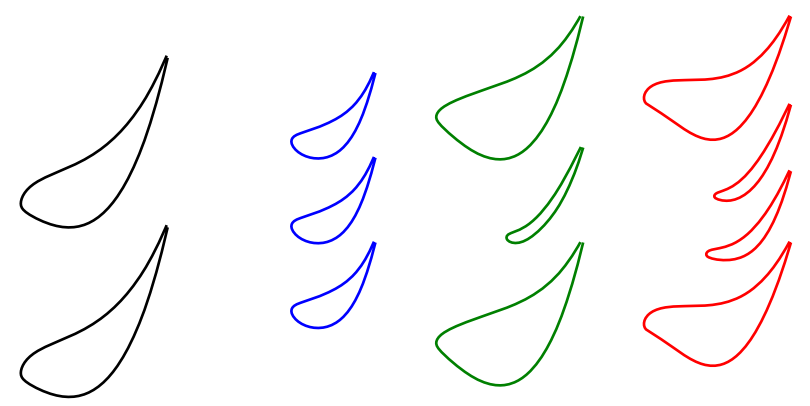
(a) NGV1
(b) NGV2
(c) NGV3
(d) NGV4

Fig. 7: NGV mid-span vane profiles 


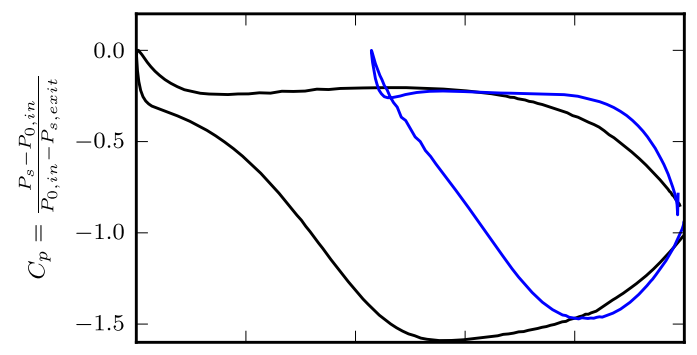

(a) NGV2

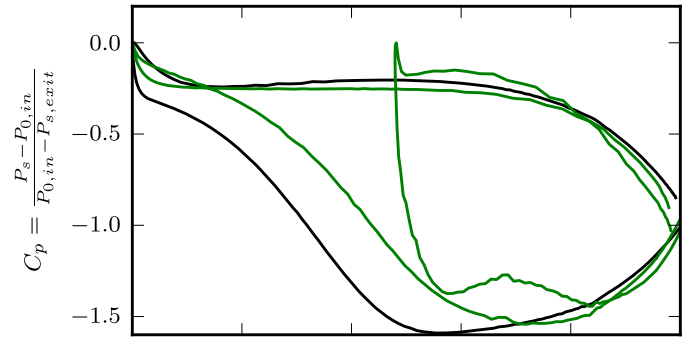

(b) NGV3

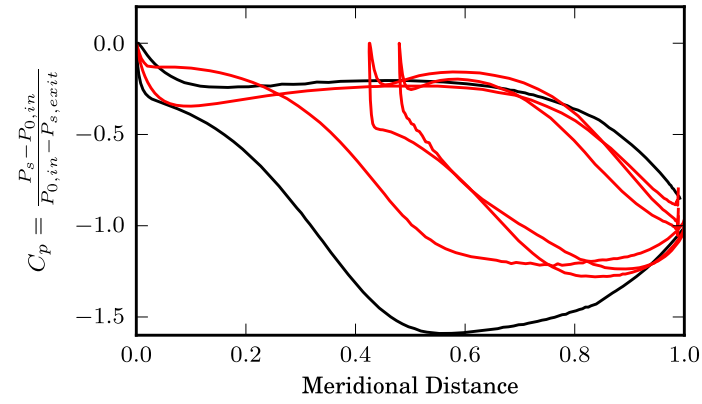

(c) NGV4

Fig. 8: NGV mid-span surface pressure distributions 


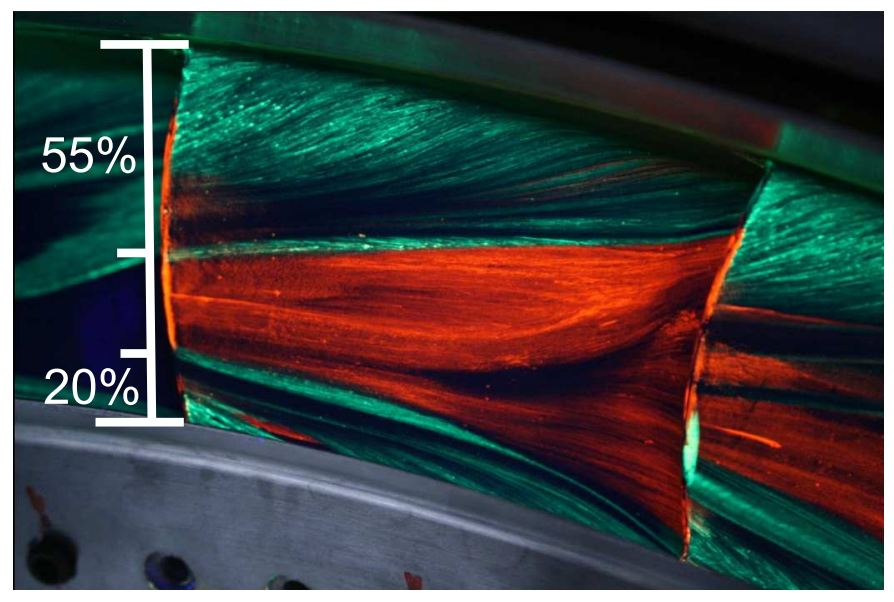

(a) NGV1

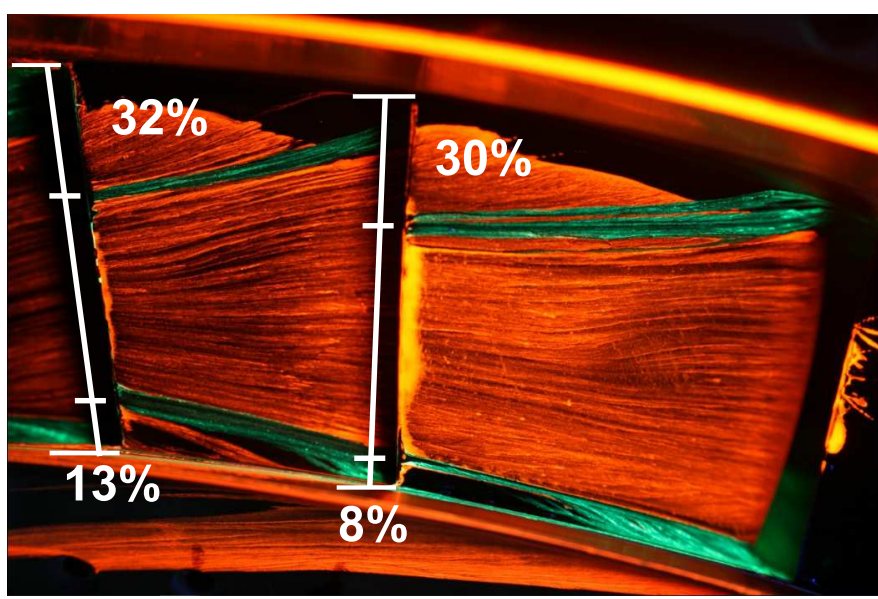

(c) NGV3

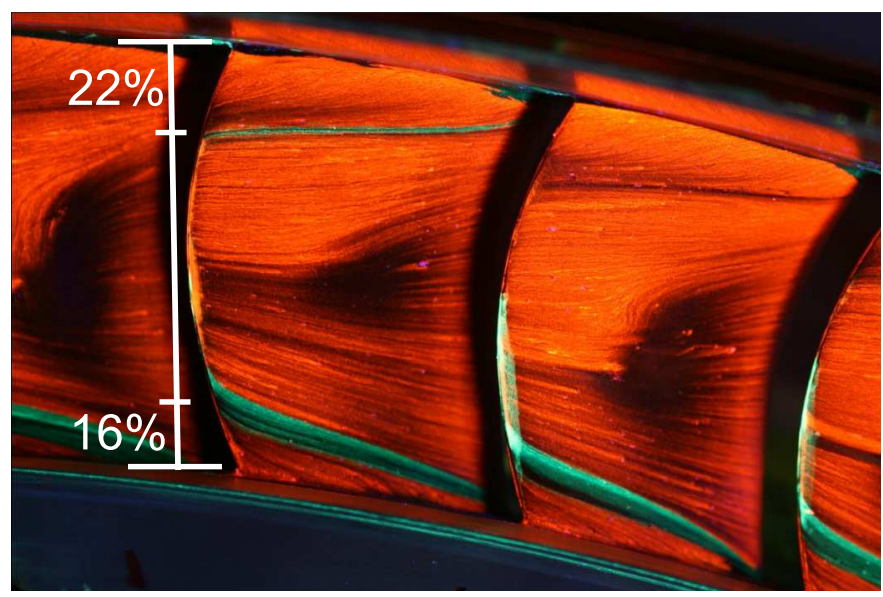

(b) NGV2

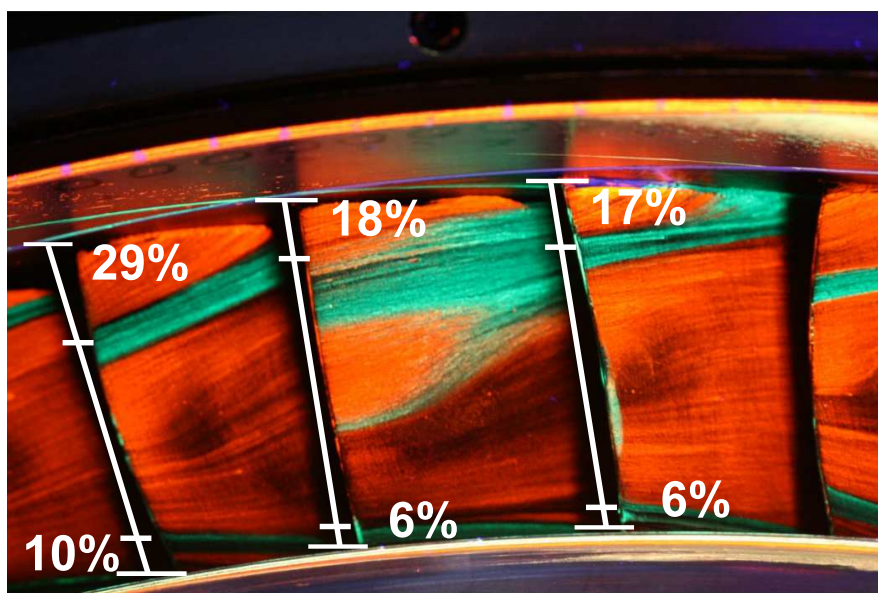

(d) NGV4

Fig. 9: Flow visualisation on the vane suction surface 

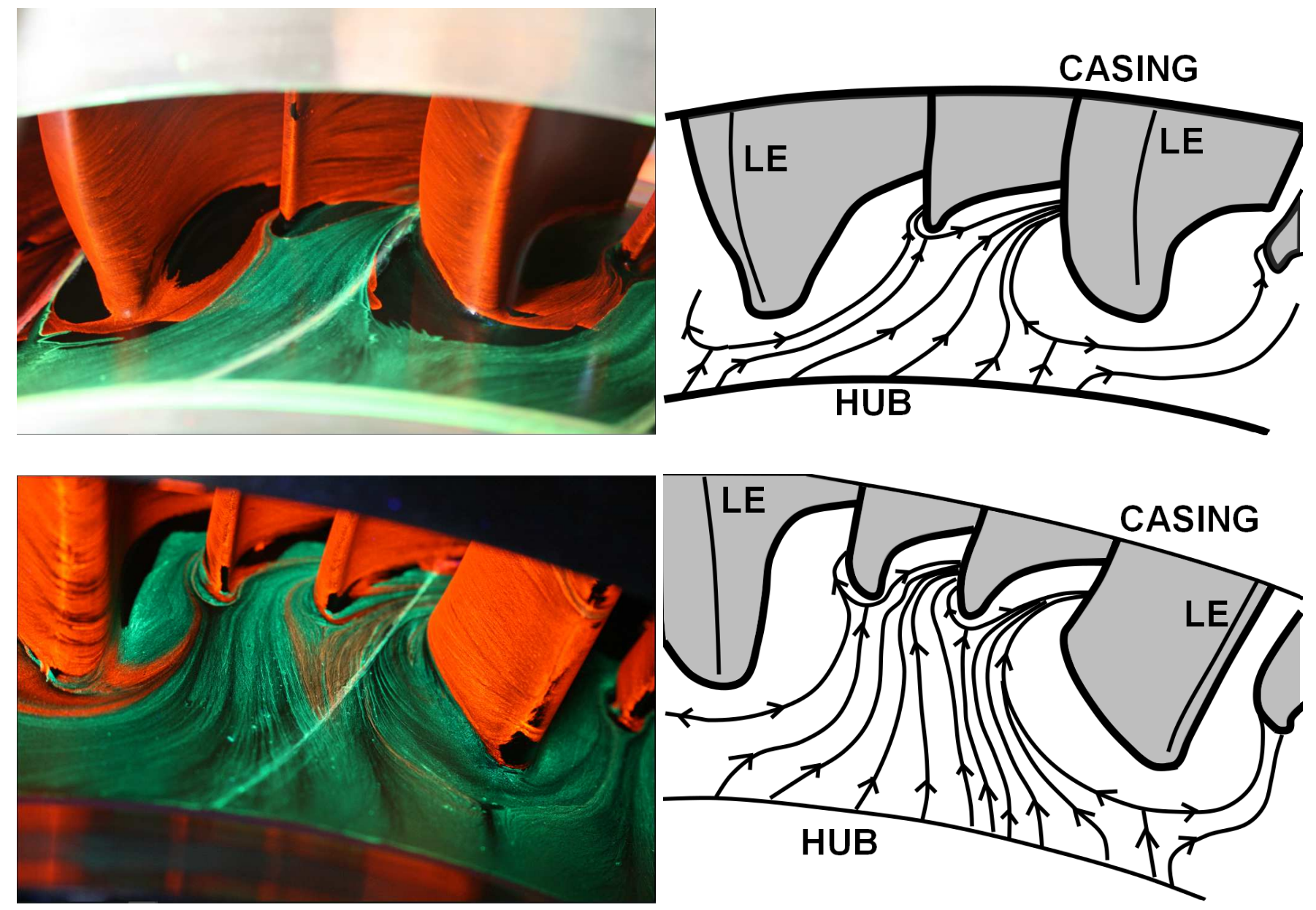

Fig. 10: Flow visualisation on the hub endwall for NGV3(upper) and NGV4(lower) 


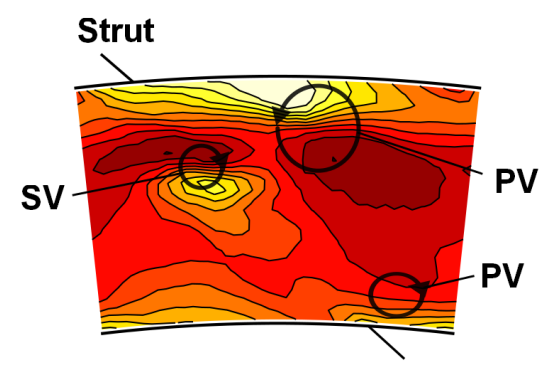

(a) NGV1

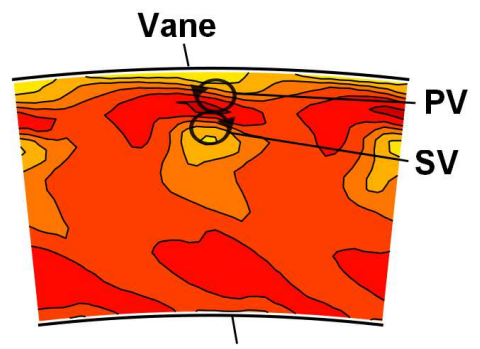

(b) NGV2

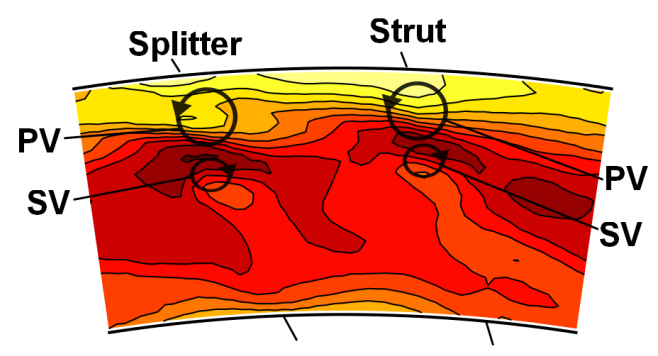

(c) NGV3

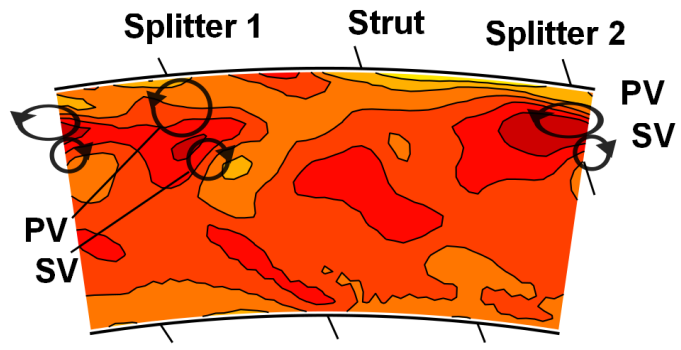

(d) NGV4

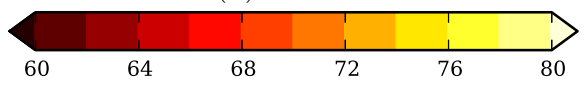

Fig. 11: NGV exit yaw angle; $\alpha_{m}=\tan ^{-1}\left(\frac{V_{t}}{V_{m}}\right)$ 


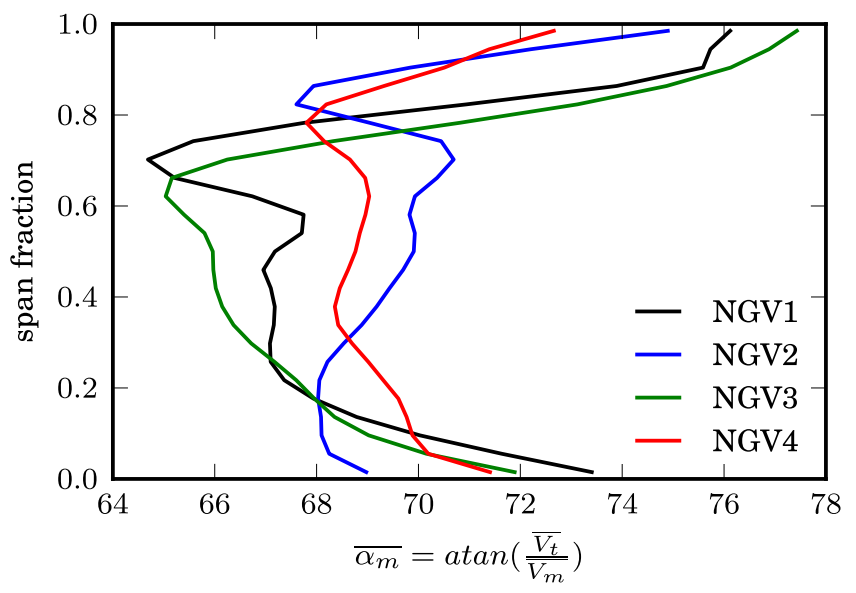

Fig. 12: NGV exit spanwise yaw angle distribution 


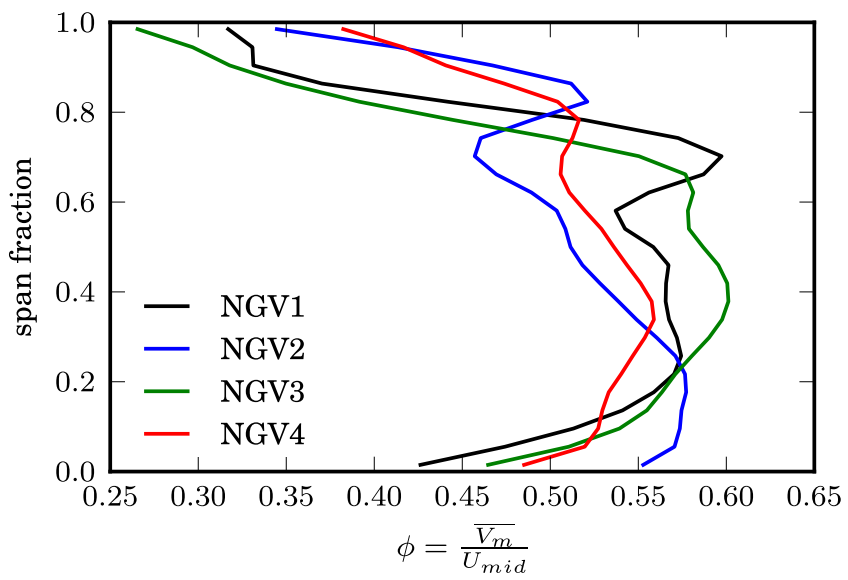

Fig. 13: NGV exit spanwise flow coefficient distribution 


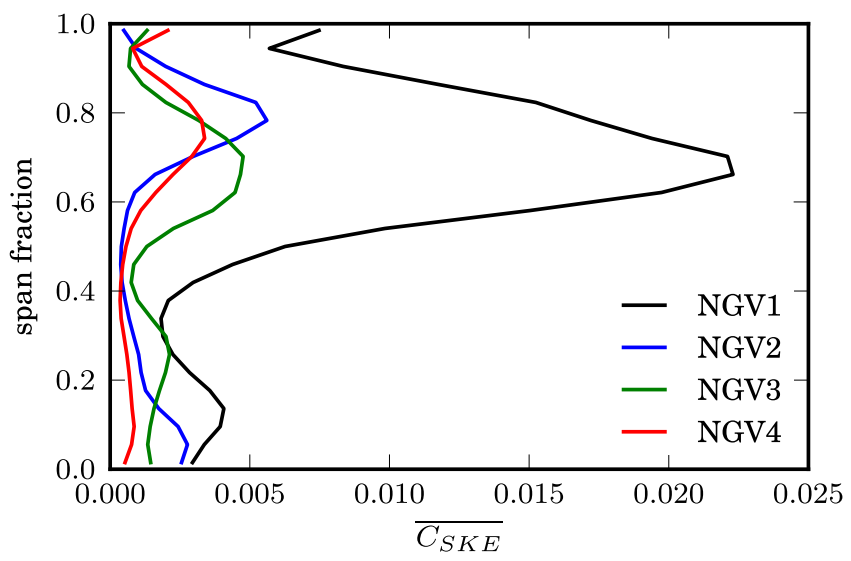

Fig. 14: NGV Exit Secondary Kinetic Energy Coefficient Distribution 


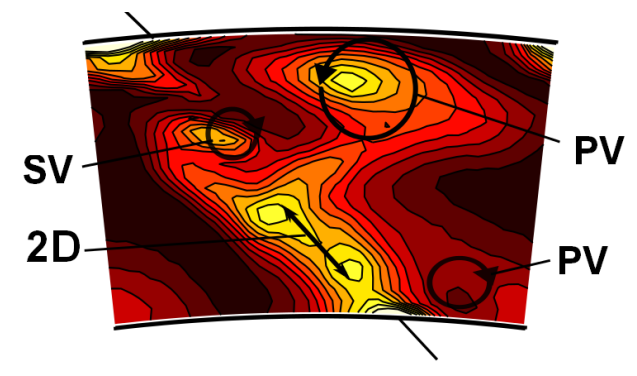

(a) NGV1

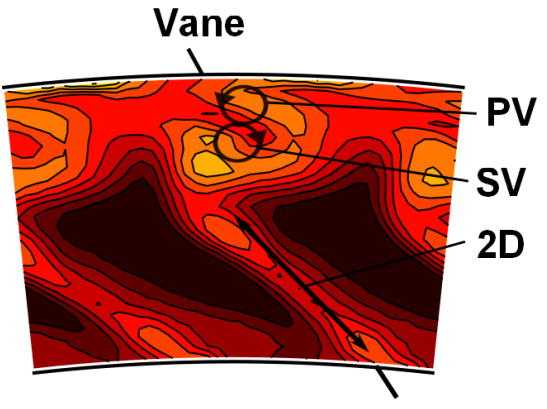

(b) NGV2

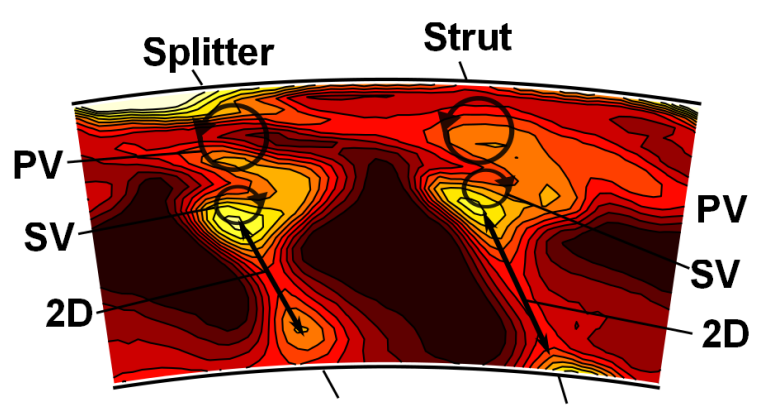

(c) NGV3

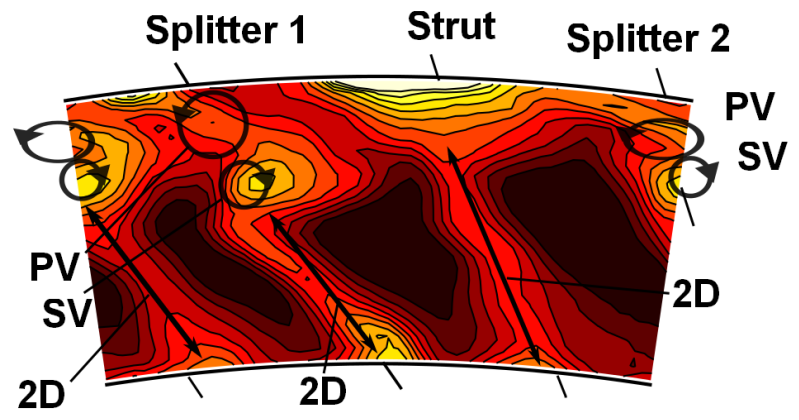

(d) NGV4

Fig. 15: Total pressure coefficient; $Y p=\frac{\overline{\overline{P_{0,1}}}-P_{0}}{\frac{1}{2} \rho\left({\overline{\overline{V_{m}}}}^{2}+{\overline{\bar{V}_{t}}}^{2}\right)}$ 


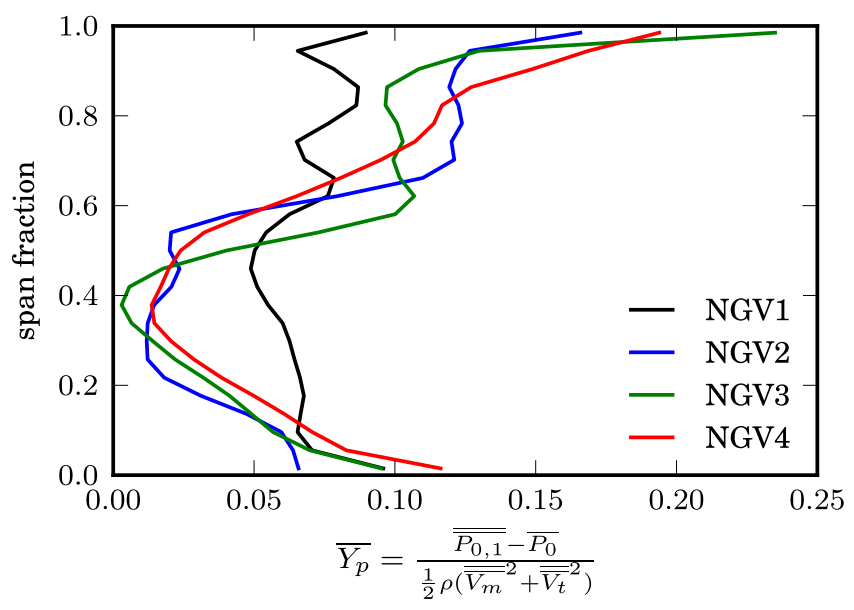

Fig. 16: NGV exit total pressure distribution 


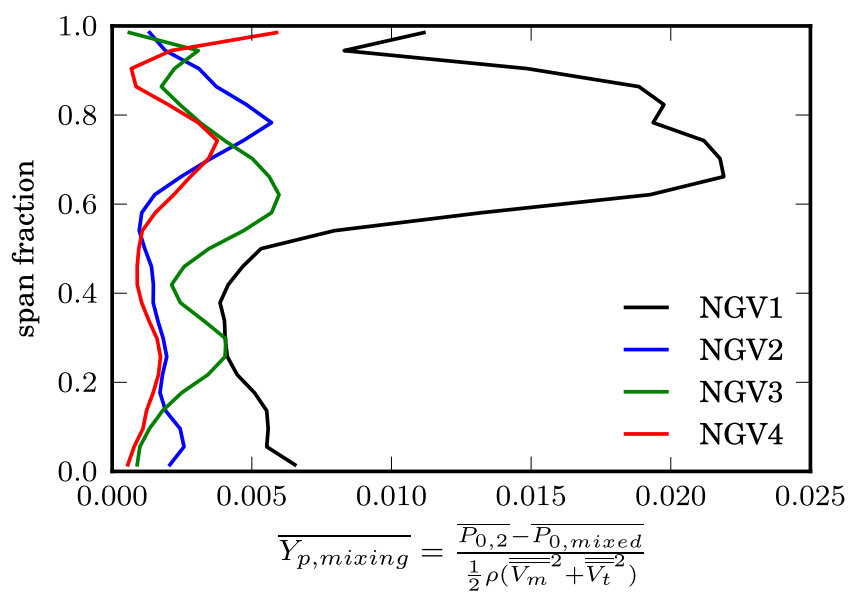

Fig. 17: NGV exit mixing loss distribution 


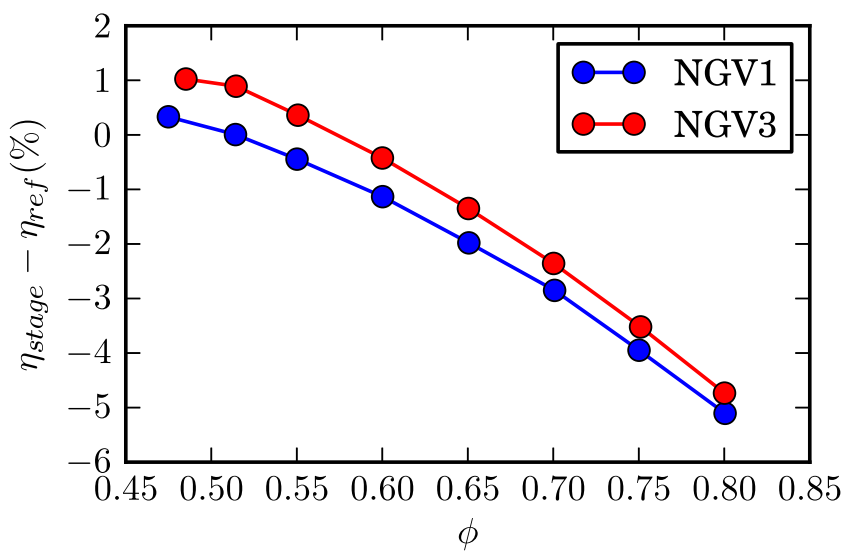

Fig. 18: Stage efficiency characteristics 


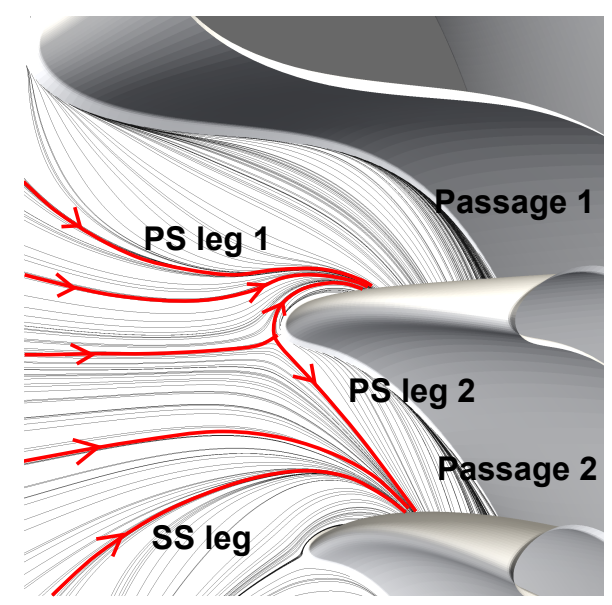

(a) NGV4

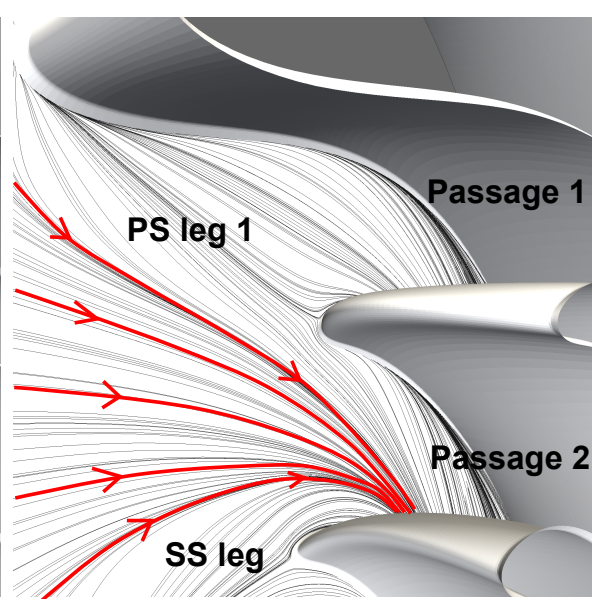

(b) $-5 \%$ chord

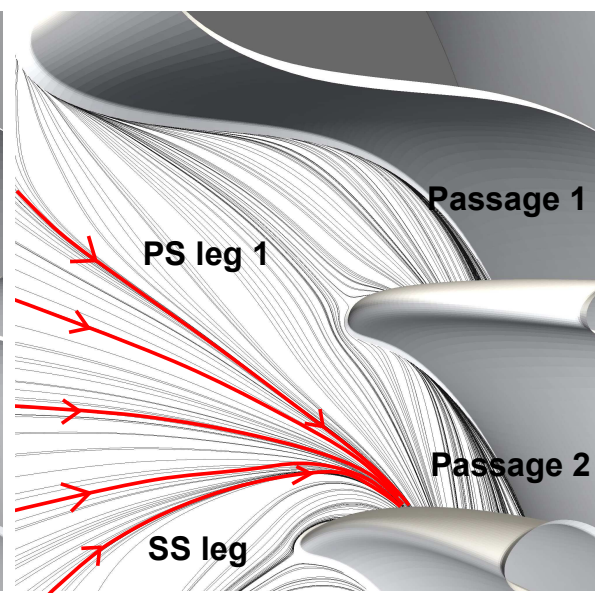

(c) $-10 \%$ chord

Fig. 19: Casing surface streamlines showing the formation of a vortex jump as the chord of the first splitter is reduced 


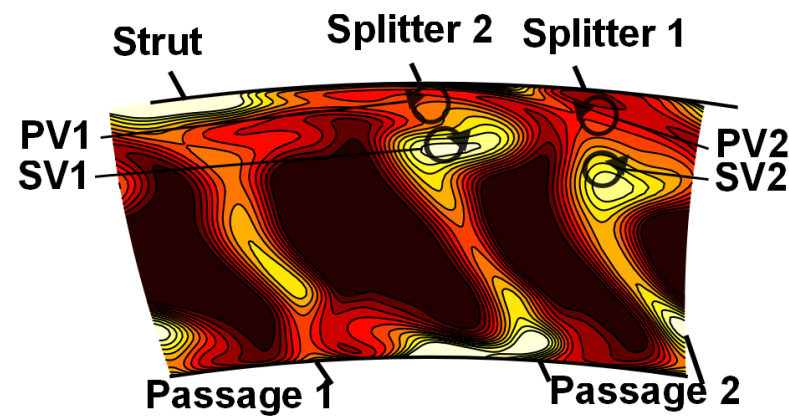

(a) NGV4

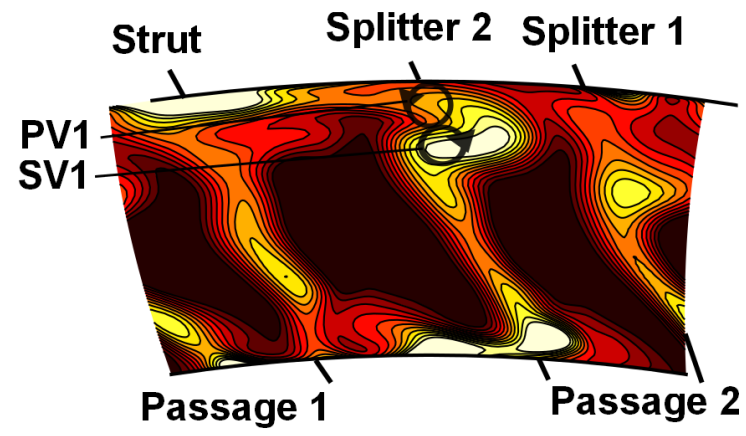

(b) $-5 \%$ chord

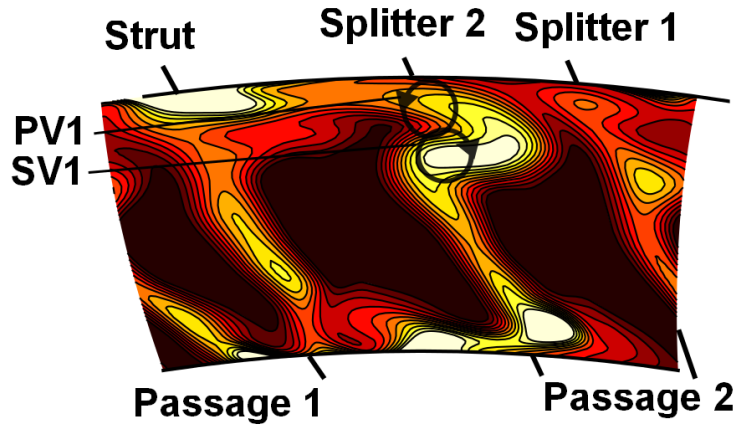

(c) $-10 \%$ chord

Fig. 20: Total pressure coefficient; $Y p=\frac{\overline{\overline{P_{0,1}}}-P_{0}}{\frac{1}{2} \rho\left({\overline{\overline{V_{m}}}}^{2}+{\overline{\bar{V}_{t}}}^{2}\right)}$ 


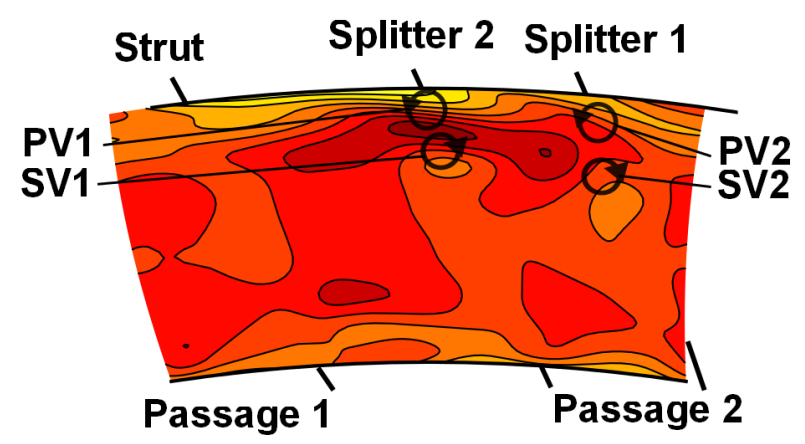

(a) NGV4

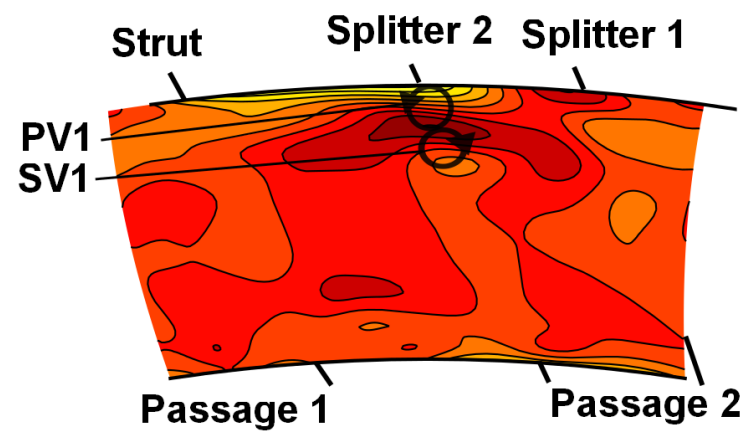

(b) $-5 \%$ chord

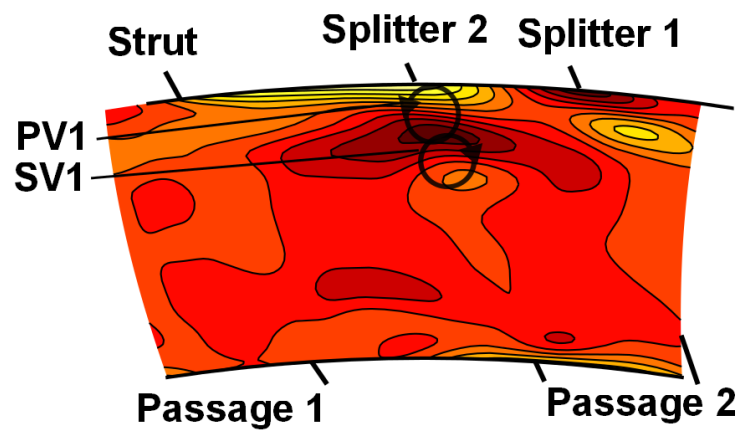

(c) $-10 \%$ chord

Fig. 21: Yaw angle: $\alpha_{m}=\tan ^{-1}\left(\frac{V_{t}}{V_{m}}\right)$ 


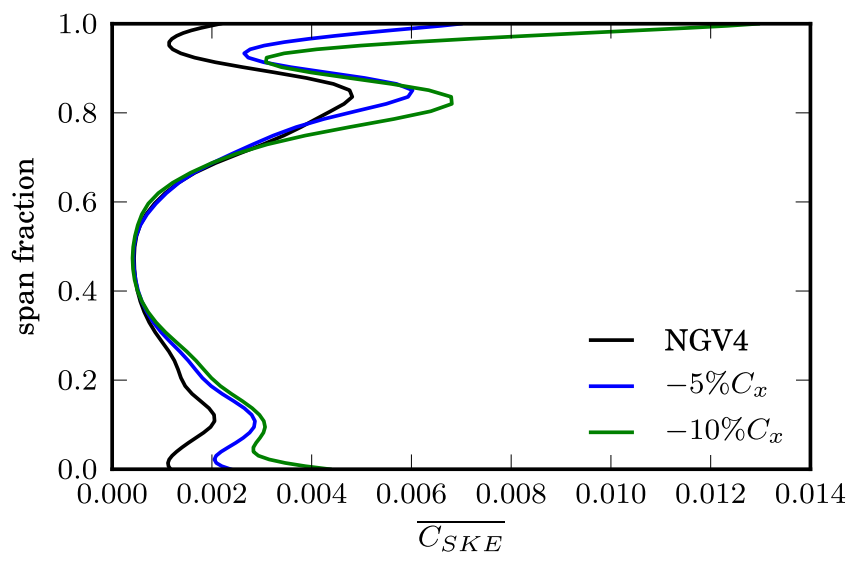

Fig. 22: NGV Exit Secondary Kinetic Energy Coefficient Distribution 